\title{
UVA Photoirradiation of Halogenated-Polycyclic Aromatic Hydrocarbons Leading to Induction of Lipid Peroxidation
}

\author{
Diógenes Herreno-Sáenz ${ }^{1 *}$, Qingsu $\mathrm{Xia}^{2}$, Li-Ting Chiu ${ }^{2}$, and Peter P. Fu ${ }^{2}$ \\ ${ }^{1}$ Department of Pharmacology and Toxicology, School of Medicine, University of Puerto Rico, P.O. Box 365067, San \\ Juan, Puerto Rico 00936-5067 and ${ }^{2}$ National Center for Toxicological Research, Jefferson, Arkansas 72079, USA \\ *Correspondence to: Dr. Diógenes Herreno-Sáenz. Email: dherreno@rcm.upr.edu
}

Received: 13 August 2005 / Accepted: 05 June 20006 / Published: 30 June 2006

\begin{abstract}
Since the finding in the 1930s, a large number of polycyclic aromatic hydrocarbons (PAHs) of different structures have been tested for potential tumorigenicity. Structure-activity relationships of halo-PAHs have been investigated to determine the regions of a PAH that may be involved in cancer initiation. From these studies, a number of halo-PAHs were found to be tumorigenic in experimental animals. It was not until the 1980s that haloPAHs were found to be present in the environment, including municipal incinerator fly ash, urban air, coal combustion, soil, snow, automobile exhausts, and tap water. Due to their widespread presence in the environment and their genotoxic activities, including carcinogenicity, many of these compounds may pose a health risk to humans. Although the biological activities, including metabolism, mutagenicity, and carcinogenicity, of haloPAHs have been studied their phototoxicity and photo-induced biological activity have not been well examined. In this study, we study the photoirradiation of a series of structure-related halo-PAHs by UVA light in the presence of a lipid, methyl linoleate, and determine as to whether or not these compounds can induce lipid peroxidation. The halo-PAHs chosen for study include 2-bromonaphthalene, 1-chloroanthracene, 9,10dibromoanthracene, 9-chlorophenanthrene, 9-bromophenanthrene, 7-chlorobenz $[a]$ anthracene, 7 bromobenz $[a]$ anthracene, $\quad$ 7-bromo-5-methylbenz $[a]$ anthracene, $\quad$ 6-chlorobenzo $[a]$ pyrene, and 6bromobenzo[ $a]$ pyrene. The results indicate that upon photoirradiation by UVA all these compounds induced lipid peroxidation at different levels. These results suggest that halo-PAHs may be harmful to human health.
\end{abstract}

\section{Introduction}

Halogenated- polycyclic aromatic hydrocarbons (halo- PAHs) are a class of compounds with one or more halogen groups attached to the aromatic rings of a polycyclic aromatic hydrocarbon (PAH) [1, 2]. The interest in halo- $\mathrm{PAH}$ compounds stems from their chemistry, including synthesis, reactions, properties, and utilization [1-6]. Since the finding in the 1930s that many PAHs are carcinogenic and are present in the environment, a large number of PAHs of different structures have been tested for potential tumorigenicity [1-3]. Structure-activity relationships of halo-PAHs have been investigated to determine the regions of a PAH that may be involved in cancer initiation $[1,2]$. From these studies, a number of halo-PAHs were found to be tumorigenic in experimental animals. It was not until the 1980s that halo-PAHs were found to be present in the environment, including municipal incinerator fly ash, urban air, coal combustion, soil, snow, automobile exhausts, and tap water [1-3,6]. Due to their widespread presence in the environment and their genotoxic activities, including carcinogenicity, many of these compounds may pose a health risk to humans. To date, the biological activities, including metabolism, mutagenicity, and carcinogenicity, of a large number of halo-PAHs have been studied [1-3, 7-15]. While people exposed to the environmental halo-PAHs on the skin are unavoidably exposed to sunlight, it is not known whether contact of halo-PAHs with concomitant exposure to sunlight would result in any deleterious effects. Consequently we report in this study the photoirradiation of a series of representative halo-PAHs that have been detected in the environment by UVA light in the presence of a lipid, methyl linoleate, and determine whether these halo-PAHs can induce lipid peroxidation. The structures, names, abbreviations, and the numberings of the halo-PAHs employed in this study are given in Figure 1, which include: 2-bromonaphthalene (2-Br-Naph), 1chloroanthracene (1-Cl-A), 9,10-dibromoanthracene $(9,10$ - 
DiBr-A), 9-chlorophenanthrene (9-Cl-Ph), 9bromophenanthrene (9-Br-Ph), 7-chlorobenz[a]anthracene (7-Cl-BA), 7-bromobenz[a]anthracene (7-Br-BA), 7bromo-5-methylbenz[a]anthracene (7-Br-5MBA), 6chlorobenzo[a]pyrene (6-Cl-BaP), and 6bromobenzo[a]pyrene (6-Br-BaP).

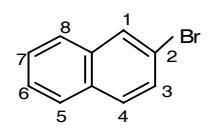

2-Bromonaphthalene (2-Br-Naph)

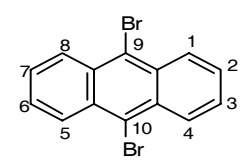

9,10-Dibromoanthracen $(9,10-\mathrm{DiBr}-\mathrm{A})$

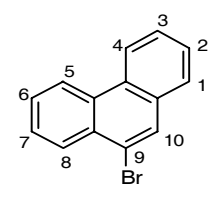

9-Bromrophenanthrene $(9-\mathrm{Br}-\mathrm{Ph})$

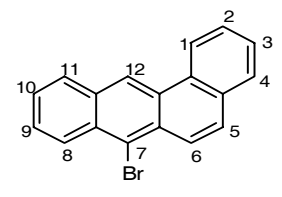

7-Bromobenz $[a]$ anthracene (7-Br-BA)

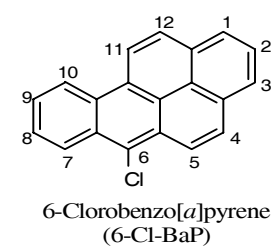

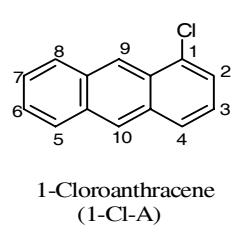
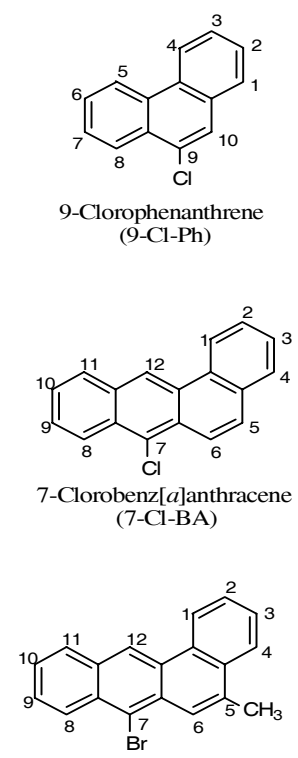

7-Bromo-5-methylbenz $[a]$ anthracene (7-Br-5MBA)

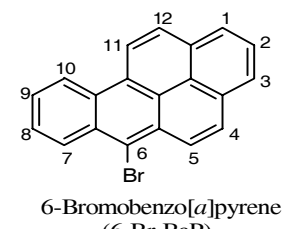

(6-Br-BaP)
Figure 1: Names and structures of the halo-PAHs used for study.

\section{Materials and Methods}

\section{Materials}

2-Bromohaphthalene, 1-Chloroanthracene, 9chlorophenanthrene, 9-bromophenanthrene, 9,10dibromoanthracene, anthracene, pheanthrene, benz $[a]$ anthracene, and benzo $[a]$ pyrene were purchased from Aldrich Chemical Co. (Milwaukee, WI). All other reagents were obtained through commercial sources and were the highest quality available. All solvents used were HPLC grade.

\section{Synthesis of Halo-PAHs}

Electrophilic aromatic substitution halogenation generally occurs by reacting PAHs with molecular halogen in the presence of Lewis acid as a catalyst, such as $\mathrm{FeCl}_{3}, \mathrm{AlCl}_{3}$, and $\mathrm{Tl}(\mathrm{OAc})_{3}[1,4,5]$. Because PAHs contain two or more aromatic rings, which can facilitate electrophilic reactions, halogenation of PAHs can sometimes proceed without the presence of a catalyst. Under mild conditions, substitution occurs at the most reactive carbon position of a $\mathrm{PAH}$, producing the kinetically controlled geometric isomer as the predominant product $[1,4,5]$. The most reactive carbon positions of benz $[a]$ anthracene and benzo $[a]$ pyrene are the C7 and C6 positions, respectively. Thus, chlorination of benz $[a]$ anthracene and benzo $[a]$ pyrene with $N$ chlorosuccinimide under mild conditions yields 7-chlorobenz $[a]$ anthracene and 6-chlorobenzo[ $a]$ pyrene, respectively. Similarly, 7-bromobenz[a]anthracene, 7bromo-5-methylbenz $[a]$ anthracene, and 6bromomethylbenzo[a]pyrene were prepared by reaction of benz $[a]$ anthracene, 5-methylbenz $[a]$ anthracene, and 6benzo $[a]$ pyrene with $N$-bromosuccinimide, respectively.

\section{Light Source}

The UVA light box was custom made with a 4-lamp unit using UVA lamps (National Biologics). The irradiance of light was determined using an Optronics OL754 Spectroradiometer (Optronics Laboratories, Orlando, FL), and the light dose was routinely measured using a Solar Light PMA-2110 UVA detector (Solar Light Inc., Philadelphia, PA). The maximum emission of the UVA is between $340-355 \mathrm{~nm}$ [16]. The light intensities at wavelengths below $320 \mathrm{~nm}$ (UVB light) and above $400 \mathrm{~nm}$ (visible light) are about two orders of magnitude lower than the maximum at $340-355 \mathrm{~nm}$.

Peroxidation of Methyl Linoleate Initiated by Photoirradiation of Halogenated Pahs and their Parent Pahs with UVA Light

Experiments were conducted with a solution of 100 $\mathrm{mM}$ methyl linoleate and $1.0 \mathrm{mM}$ substrate in methanol. Samples were placed in a UV-transparent cuvette and irradiated with 0,7 , and $21 \mathrm{~J} / \mathrm{cm} 2$ of UVA light. After irradiation, the methyl linoleate hydroperoxide products were separated by HPLC using a Prodigy $5 \mathrm{~m}$ ODS column (4.6 x $250 \mathrm{~mm}$, Phenomenex, Torrance, CA) eluted isocratically with $10 \%$ water in methanol (v/v) at $1 \mathrm{~mL} / \mathrm{min}$. The levels of lipid peroxidation were measured by calculation of the amount of methyl linoleate hydroperoxides from the HPLC peak area by monitoring the elution at $235 \mathrm{~nm}$ following the method of Tokita [17].

\section{Results and Discussion}

\section{Photoirradiation of Representative Halo-PAHs}

Ten representative parent halo-PAHs were selected for the study of photoirradiation with UVA in the presence of a lipid, methyl linoleate. As shown in Figure 1, these halo-PAHs include: 2-bromonaphthalene (2-Br-Naph), 1chloroanthracene (1-Cl-A), 9,10-dibromoanthracene (9,10-DiBr-A), 9-chlorophenanthrene (9-Cl-Ph), 9bromophenanthrene (9-Br-Ph), 7-chlorobenz[a]anthracene (7-Cl-BA), 7-bromobenz[a]anthracene (7-Br-BA), 7- 
bromo-5-methylbenz[a]anthracene (7-Br-5MBA), 6chlorobenzo[a]pyrene (6-Cl-BaP), and 6bromobenzo[a]pyrene (6-Br-BaP). The halo-PAHs possess different sizes, ranging from two to five benzo-rings, and exhibit various carcinogenic potencies [2] (Table 1). For comparison, their parent PAHs, anthracene, phenanthrene, benz $[a]$ anthracene (BA), 5-methylbenz[a]anthracene (5MBA), and benzo[ $a]$ pyrene (BaP) are included for study. Each of the halo-PAHs and parent PAHs received two light doses, 7 and $21 \mathrm{~J} / \mathrm{cm}^{2}$, respectively.

The resulting photoirradiation products were separated and analyzed by reversed HPLC. Following the method of Tokita [17], the levels of lipid peroxidation from each experiment were measured by calculation of the amount of methyl linoleate hydroperoxides from the HPLC peak area by monitoring the elution at $235 \mathrm{~nm}$. Upon calculations of the HPLC peak areas, the levels of induction of lipid peroxidation of the halo-PAHs and PAHs were identified (Table 1). In general, each of the halo-PAHs and PAHs received two light doses, 7 and $21 \mathrm{~J} / \mathrm{cm}^{2}$, respectively, and the resulting lipid peroxidation was found to be dosedependent (Table 1). Among the tested PAHs, phenanthrene was the only PAH that did not induce lipid peroxidation in considerable amount. The levels of lipid peroxidation induction by these $\mathrm{PAH}$ are in the order: $\mathrm{BaP}>\mathrm{BA}>$ anthracene $>$ 5-MBA > phenanthrene. Compared with their reported tumorigenicity potency [2], there is no correlation between the photo-induced lipid peroxidation and tumorgenicity.

Table 1: Induction lipid peroxidation by halo-PAHs with concomitant exposure to UVA light irradiation ${ }^{\mathrm{a}}$

\begin{tabular}{|c|c|c|c|}
\hline Compound & $0 \mathrm{~J} / \mathrm{cm}^{2}$ & $7 \mathrm{~J} / \mathrm{cm}^{2}$ & $21 \mathrm{~J} / \mathrm{cm}^{2}$ \\
\hline Methyl linoleate (ML) & 145 & $212 \pm 34$ & $267 \pm 47$ \\
\hline 2-Br-Naph & 137 & $402 \pm 49$ & $1135 \pm 93$ \\
\hline Anthracene (A) & 138 & $1350 \pm 66$ & $2459 \pm 197$ \\
\hline $1-\mathrm{Cl}-\mathrm{A}$ & 122 & $1976 \pm 100$ & $3759 \pm 218$ \\
\hline 9,10-DiBr-A & 137 & $2260 \pm 150$ & $5144 \pm 330$ \\
\hline Phenanthrene $(\mathrm{Ph})$ & 144 & $321 \pm 13$ & $803 \pm 37$ \\
\hline 9-Cl-Ph & 138 & $645 \pm 22$ & $1301 \pm 100$ \\
\hline 9-Br-Ph & 134 & $537 \pm 27$ & $721 \pm 58$ \\
\hline Benz $[a]$ anthracene (BA) & 141 & $2719 \pm 133$ & $3917 \pm 403$ \\
\hline 7-Cl-BA & 119 & $2540 \pm 179$ & $3640 \pm 225$ \\
\hline 7-Br-BA & 137 & $2494 \pm 301$ & $5309 \pm 638$ \\
\hline $5 \mathrm{MBA}$ & 142 & $1582 \pm 58$ & $1885 \pm 236$ \\
\hline 7-Br-5MBA & 115 & $2296 \pm 112$ & $3769 \pm 216$ \\
\hline Benzo $[a]$ pyrene $(\mathrm{BaP})$ & 100 & $2467 \pm 217$ & $5819 \pm 425$ \\
\hline 6-Cl-BaP & 153 & $2404 \pm 29$ & $4840 \pm 137$ \\
\hline 6-Br-BaP & 135 & $2639 \pm 224$ & $5201 \pm 291$ \\
\hline
\end{tabular}

${ }^{a}$ Average of three Experiments/standard deviation

\section{Comparison of Level of Lipid Peroxidation Formation}

\section{Anthracene Series}

Upon photoirradiation, anthracene, 1-chloroanthracene, and 9,10-dimethylanthracene all induced lipid peroxidation, with 9,10-dimethylanthracene most effectively (Figure 2).

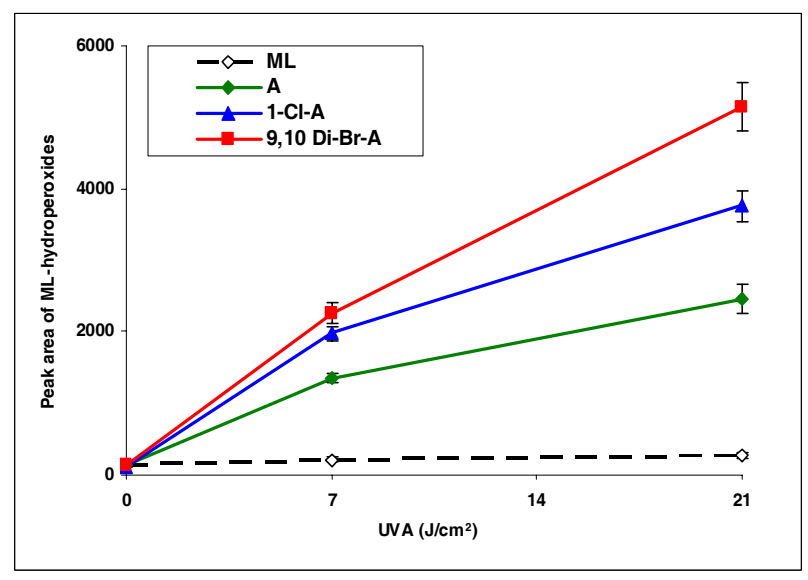

Figure 2: Peroxidation of methyl linoleate initiated by anthracene, 1-Cl-anthracene and 9,10-DiBr-anthracene with, 0,7 , and $21 \mathrm{~J} / \mathrm{cm} 2$ of UVA light.

\section{Phenenthrene Series}

This series is different from the anthracene series. Among phenanthrenes (9-chlorophenanthrene and 9bromophenanthrene), 9-chlorophenanthrene exhibited strong induction of lipid peroxidation, while the other compounds were weak inducers (Figure 3).

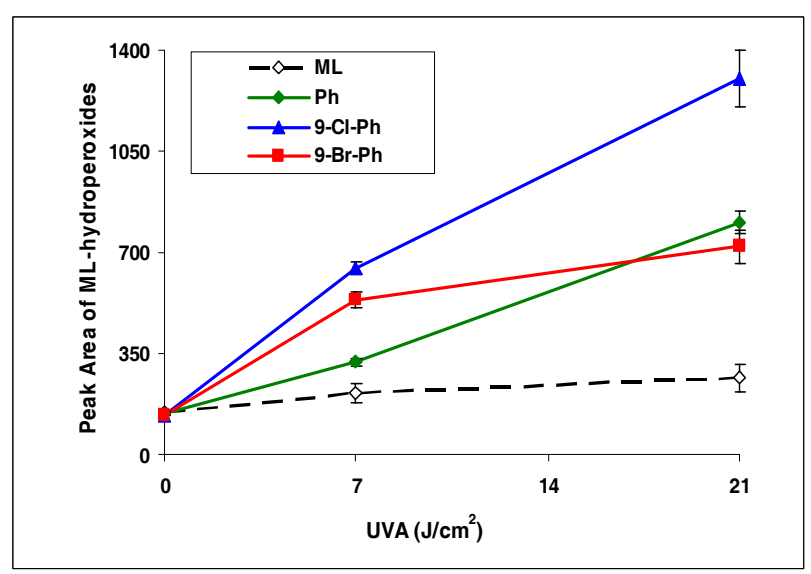

Figure 3: Peroxidation of methyl linoleate initiated by phenanthrene , 9-Cl-phenanthrene , 9-Br-phenanthrene, with, 0,7 , and $21 \mathrm{~J} / \mathrm{cm} 2$ of UVA light.

\section{Benz[a]anthracene Series}

BA, 7-Cl-BA, and 7-Br-BA are strong inducers (Figure 4). 5-Methylbenz[a]anthracene, with a methyl group attached to the BA molecule, induced lipid peroxidation as about half of that by BA (Figure 5). However, with an additional bromo group at the $\mathrm{C} 7$ position, the resulting compound 7-Br-5-MBA induced lipid peroxidation at a level similar to that by BA. 


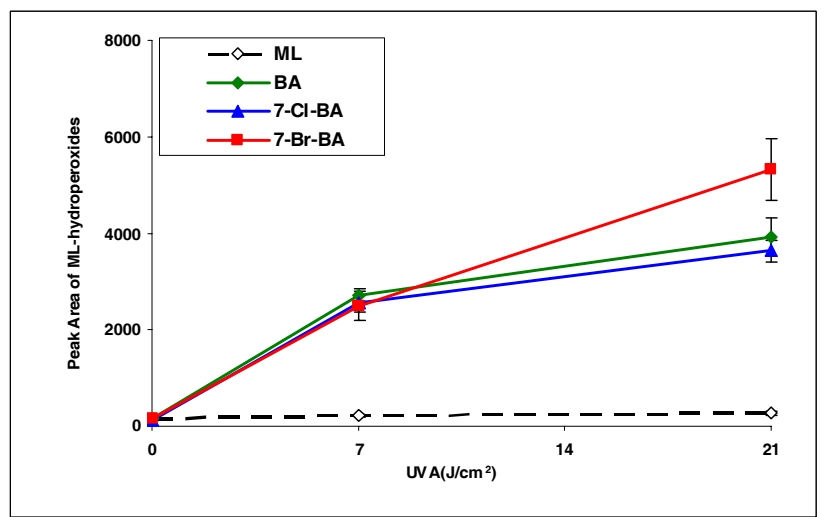

Figure 4: Peroxidation of methyl linoleate initiated by benzo[ $[a]$ pyrene, 7-Cl-benzo[ $[a]$ pyrene, 7-Brbenzo[ $a]$ pyrene, with, 0,7 , and $21 \mathrm{~J} / \mathrm{cm} 2$ of UVA light.

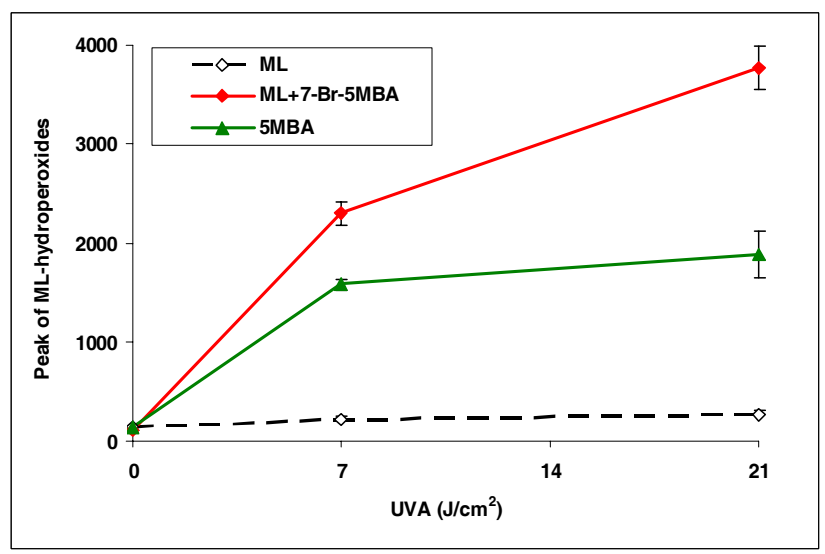

Figure 5: Peroxidation of methyl linoleate initiated by 5methylbenz[a]anthracene and 7-bromo-5methylbenz $[a]$ anthracene with, 0,7 , and $21 \mathrm{~J} / \mathrm{cm} 2$ of UVA light.

\section{Benzo[a]pyrene Series}

Among all the halo-PAHs and PAHs used in this study, $\mathrm{BaP}$, 6-Cl-BaP, and 6-Br-BaP induced lipid peroxidation at the highest levels (Figure 6). However, there were no significant differences among these three compounds in induction of lipid peroxidation.

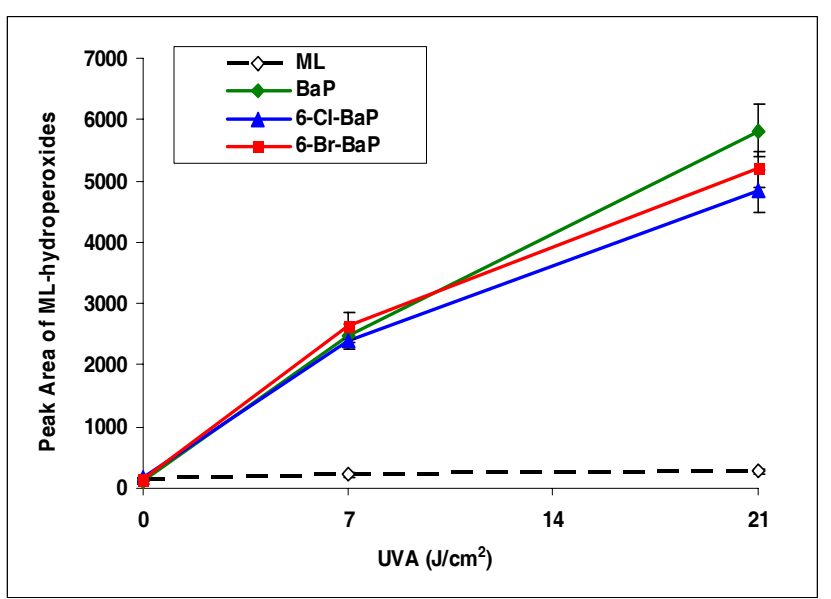

Figure 6: Peroxidation of methyl linoleate initiated by benzo[a]pyrene, 6-Cl-benzo $[a]$ pyrene, 6-Brbenzo[a]pyrene, with, 0,7 , and $21 \mathrm{~J} / \mathrm{cm} 2$ of UVA light.

\section{Conclusion}

1. Upon photoirration with UVA light, all the haloPAHs used in this study induced lipid peroxidation, but with different induction potencies.

2. The levels of lipid peroxidation by each halo-PAHs and PAHs were dose (light) dependent.

3. In general, the addition of a halogen atom to the parent $\mathrm{PAH}$ resulted in lipid peroxidation either increase, decrease or no effect on the capability of induction of lipid peroxidation.

4. There was no correlation between the level of lipid peroxidation and the tumorigenic potency of the compounds studied.

5. Halo-PAHs are environmental contaminants and, as shown in our study, can induce lipid peroxidation, these compounds may pose adverse health effect in humans.

\section{References}

1. Fu, P. P.; Von Tungeln, L. S.; Chiu L.-H.; Own Z. Y.: Halogenateo-polycyclic aromatic hydrocarbons: a class of genotoxic environmental pollutants. Environ. Carcinogen. Ecotoxicol. Rev., 1999, C17(2), 71-109.

2. Dipple, A.; Moschel, R. C.; Bigger, C. A. H.: in: Chemical Carcinogens, edited by C. E. Searle, Monograph 182, American Chemical Society, Washington, DC, 1984. pp. 41.

3. Hartwell, J. L.: Survey of Compounds Which Have Been Tested for Carcinogenic Activity, U.S. Public Health Service Publication No. 149, Washington, D.C., 1951.

4. Clar, E.: Polycyclic Hydrocarbons, Academic Press, New York, NY, 1964, Vol. 1 and 2.

5. Harvey, R. G.: Polycyclic Aromatic Hydrocarbons, Wiley-VCH, Inc., 1997.

6. Thompson, J. I. \& Company: Survey of Compounds Which Have Been Tested for Carcinogenic Activity, U.S. Public Health Service Publication No. 149, Washington, D.C., 1968-1969.

7. Fu, P. P.; Yang, S. K.: Stereoselective metabolism of 7-bromobenz[a]anthracene by rat liver microsomes: absolute configuration of

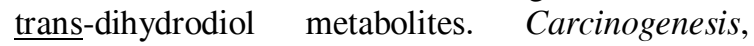
1983, 4, 979-984.

8. Chiu, P.-L.; Fu, P. P.; Yang, S. K.: Stereoselectivity of rat liver microsomal enzymes in the metabolism of 7-fluorobenz[a]anthracene and mutagenicity of metabolites. Cancer Res. 1984, 44, 562-570.

9. Fu, P. P.; Von Tungeln L. S.; Unruh, L. E.; Ni, Y.-C; Chou, M. W.: Comparative regioselective and stereoselective metabolism of 7-chlorobenz[a]anthracene and 7-bromobenz[a]anthracene by mouse and rat liver microsomes. Carcinogenesis, 1991, 12, 371-378.

10. Xiao, Y.; Von Tungeln, L. S.; Chou, M. W.; Hart, R. W.; Fu, P. P.: Effect of caloric restriction on the metabolism of 7-bromobenz[a]anthracene and 7-fluorobenz[a]anthracene by male $\mathrm{B} 6 \mathrm{C} 3 \mathrm{~F}_{1}$ mouse liver microsomes: Reduction of metabolic activation pathway. Age, 1993, 16(4), 160-165. 
11. Xiao, Y.; Von Tungeln, L. S.; Chou, M. W.; Hart, R. W.; Fu, P. P.: Effect of caloric restriction on the stereoselective metabolism of

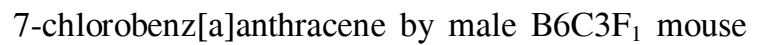
liver microsomes. Age, 1993, 16(4), 166-172.

12. Own, Z. Y.; Chiu, L.-H.; Von Tungeln, L. S.; Deck, S. J.; Vingiello, F. A.; Fu, P. P.: Synthesis and rat liver microsomal metabolism of 2chlorodibenz[a,l]pyrene and 10-chlorodibenz[a,l]pyrene. Polycyclic Aromat. Cpds. 1996, 11, 333-340.

13. Fu, P.P.;Von Tungeln, L.S.; Zhan, D.-J.; Bucci, T. Potent tumorigenicity of 7-chlorobenz[a]anthracene and 7-bromobenz[a]anthracene in the neonatal B6C $3 F_{1}$ male mouse. Cancer Lett. 1996,101, 37-42.

14. Xia, Q.; Yi P.; Zhan, D.-J.; Von Tungeln, L. S.; Hart, R. W.; Heflich, R. H.; Fu, P. P.: Liver tumors induced in $\mathrm{B}_{6} \mathrm{C}_{3} \mathrm{~F}_{1}$ mice by 7chlorobenz[a]anthracene bromobenz[a]anthracene contain K-ras oncogene mutations, Cancer Letters, 1998, 123, 21-25.

15. Hong, F.; Von Tungeln, L. S.; Fu, P. P.; Watson F.: Stereoselective metabolism of anthracene, 9-methylanthracene, 9,10-dimethylanthracene, 9-chloroanthracene, and 9-nitroanthracene by liver microsomes of 15-day-old male $\mathrm{B}_{6} \mathrm{C} 3 \mathrm{~F}_{1}$ mice. Polycyclic Aromat. Cpd., 1999, 16, 235-244.

16. Cherng, S.; Xia, H. Q.; Blankenship, L. R.; Freeman J. P.; Wamer, W. G.; Howard, P.C.; Fu, P.P. Photodecomposition of retinyl palmitate in ethanol by UVA light - formation of photodecomposition products, reactive oxygen species, and lipid peroxides. Chem. Res. Toxicol., 2005, 18,129-138.

17. Totika, M.: Identification of new geometric isomers of methyl linoleate hydroperoxide and their chromatographic behavior. Biosci. Biotechnol. Biochem, 2000, 64, 1044-1056. 